\section{Targeting CD47/TNFAIP8 by miR-155 overcomes drug resistance and inhibits tumor growth through induction of phagocytosis and apoptosis in multiple myeloma}

\author{
Nasrin Rastgoo, ${ }^{1}$ Jian Wu, ${ }^{1}$ Aijun Liu, ${ }^{2}$ Maryam Pourabdollah, ${ }^{1}$ \\ Eshetu G. Atenafu, ${ }^{3}$ Donna Reece, ${ }^{4}$ Wenming Chen ${ }^{2}$ and Hong Chang ${ }^{1,2}$ \\ ${ }^{1}$ Department of Laboratory Medicine and Pathobiology, University of Toronto, Toronto, \\ Ontario, Canada; ${ }^{2}$ Department of Hematology, Beijing Chaoyang Hospital, Capital \\ University Beijing, Beijing, China; ${ }^{3}$ Department of Biostatistics, University Health \\ Network, Toronto, Ontario, Canada and ${ }^{4}$ Department of Hematology and Medical \\ Oncology, University Health Network, Toronto, Ontario, Canada
}

\section{ABSTRACT}

T he mechanisms of drug resistance in multiple myeloma (MM) are poorly understood. Here we show that CD47, an integrin-associated receptor, is significantly up-regulated in drug resistant myeloma cells in comparison with parental cells, and that high expression of CD47 detected by immunohistochemistry is associated with shorter progression-free and overall survivals in MM patients. We show that miR-155 is expressed at low levels in drug resistant myeloma cells and is a direct regulator of CD47 through its 3'UTR. Furthermore, low miR-155 levels are associated with advanced stages of disease. MiR-155 overexpression suppressed CD47 expression on myeloma cell surface, leading to induction of phagocytosis of myeloma cells by macrophages and inhibition of tumor growth. MiR-155 overexpression also re-sensitized drug-resistant myeloma cells to bortezomib leading to cell death through targeting TNFAIP8, a negative mediator of apoptosis in vitro and in vivo. Thus, miR-155 mimics may serve as a promising new therapeutic modality by promoting phagocytosis and inducing apoptosis in patients with drug-refractory/relapsed MM.

\section{Introduction}

Multiple myeloma (MM) is a plasma cell malignancy characterized by abnormal proliferation of clonal plasma cells in the bone marrow (BM). ${ }^{1}$ Current therapies, such as the proteasome inhibitor bortezomib (BTZ), have improved the outcome of patients. Nevertheless, MM remains an incurable disease with a high rate of relapse and development of drug resistance. ${ }^{2}$ So far, the pathogenic mechanisms underlying drug resistance of MM have not been fully elucidated. Ubiquitously expressed CD47 is known to block phagocytosis by binding to signal regulatory protein $\alpha$ (SIRP $\alpha$ ) on macrophages. This receptor-ligand binding can also inhibit initiation of an innate immune response. ${ }^{3,4}$ High expression of CD47 is associated with tumor growth, metastasis, recurrence, or drug resistance in hematologic malignancies. ${ }^{5.7}$ In plasma cell neoplasms, transition from monoclonal gammopathy of undetermined significance (MGUS) to multiple myeloma (MM) has been associated with a significant increase in CD47 expression. ${ }^{8}$ It has also been shown that CD47 is expressed in different sub-populations of peripheral blood mononuclear cells (PBMC); however, its highest expression is observed in MM cells and it is significantly increased in the presence of a tumor microenvironment. ${ }^{9}$ In addition, CD47 plays a role in bone resorption in $\mathrm{MM}$ by inducing macrophage fusion and osteoclast formation. ${ }^{10}$ However, the clinical relevance of CD47 expression in MM patients and the role of CD47 upregulation in MM drug resistance have not yet been established.

In the current study, we examined the expression of CD47 in MM patients' cells and assessed its correlation with clinical outcomes. Moreover, to gain an insight into regulatory mechanisms underlying CD47 high expression in MM, we sought to explore if miRNAs could be involved. This is supported by the fact that miRNAs play an important role in the pathogenesis of MM. Particularly, deregulated miRNAs can
Haematologica 2020
Volume 105(12):2813-2823

\section{Correspondence:}

HONG CHANG

hong.chang@uhn.on.ca

Received: May 21, 2019.

Accepted: November 27, 2019.

Pre-published: November 28, 2019.

doi:10.3324/haematol.2019.227579

(C)2020 Ferrata Storti Foundation

Material published in Haematologica is covered by copyright. All rights are reserved to the Ferrata Storti Foundation. Use of published material is allowed under the following terms and conditions:

https://creativecommons.org/licenses/by-nc/4.0/legalcode. Copies of published material are allowed for personal or internal use. Sharing published material for non-commercial purposes is subject to the following conditions: https://creativecommons. org//icenses/by-nc/4.0/legalcode, sect. 3. Reproducing and sharing published material for commercial purposes is not allowed without permission in writing from the publisher. 
influence gene-expression and functional responses of $\mathrm{MM}$ cells. ${ }^{11}$ Importantly, miRNAs are now considered as a new class of agents for therapeutic intervention. ${ }^{12}$ Here, we found that the tumor suppressor miRNA, miR-155, directly targeted CD47 and was under-expressed in drug resistant MM cells. Restoration of miR-155 in drug resistant MM cells induced phagocytosis of MM cells by macrophages. Moreover, miR-155 overexpression sensitized MM resistant cells to bortezomib by targeting TNF- $\alpha$-induced protein 8 (TNFAIP8). TNFAIP8 acts as a negative mediator of apoptosis and may play a role in tumor progression. It suppresses the TNF-mediated apoptosis by inhibiting caspase-8 activity but not the processing of procaspase- 8 , subsequently resulting in inhibition of BID cleavage and caspase-3 activation. ${ }^{13-15}$ The negative correlation between miR-155 and its targets, CD47 and TNFAIP8, is significantly associated with disease progression in MM patients. Thus, the dysregulation of miR-155/CD47/TNFAIP8 axis contributes to drug resistance and represents a new therapeutic target for MM.

\section{Methods}

\section{Patients}

A total of 74 cases diagnosed with MM were included in this study. The selection criteria were availability of the clinical/laboratory data and pathologic specimens as well as receiving the same treatment protocol: 4-5 cycles of vincristine, Adriamycin and dexamethasone as induction and one course of melphalan $200 \mathrm{mg} / \mathrm{m}^{2}$ followed by autologous stem cell transplant (ASCT). The BM specimens used for staining were obtained before starting any treatment. There were 42 males and 32 females with median age of 55 (range: 34-73) years. Median follow-up time after transplant was 4.91 (range: 0.20-15.94) years (Online Supplementary Table S1). This study was approved by the research ethics committee of University Health Network, Toronto, in accordance with the Declaration of Helsinki.

\section{Myeloma cell lines and primary multiple myeloma samples}

The MM parental cell lines (RPMI-8226 and MM.1S) and MM.1R, which is resistant to dexamethasone, were obtained from ATCC. RPMI-8226-R5, a multidrug-resistant MM cell line that is cross-resistant to BTZ, was kindly provided by Dr. R Buzzeo. ${ }^{16}$ Resistance of RPMI-8226-R5 and MM.1R to the proteasome inhibitors BTZ and MG132 had been shown in our previous study. ${ }^{17}$ All cell lines were cultured in complete RPMI-1640 medium supplemented with $10 \%$ FBS, as described previously. ${ }^{18}$ $\mathrm{CD} 138^{+}$cells were freshly isolated and purified from the bone marrow of MM patients and normal healthy donors.

Details of immunohistochemical (IHC) staining, cell culture and generation of stable cell lines, cytotoxicity and apoptosis assay, immunostaining cell surface targets and indirect flow cytometry, luciferase reporter assay, miRNA mimics transfection, real-time polymerase chain reaction (RT-PCR), phagocytosis assay, animal xenograft model studies and statistical analysis are all available in the Online Supplementary Methods.

\section{Results}

CD47 is increased in multiple myeloma and its overexpression correlated with disease progression and poor survival of multiple myeloma patients

To investigate the potential clinical significance of
CD47 expression in MM, we evaluated CD47 protein expression in a cohort of 74 newly diagnosed MM patients by immunohistochemical analysis on consecutive tissue sections of CD138 positive myeloma cells (Figure 1A). The results showed that low (30-155) and high (160-240) H-score (Online Supplementary Figure S1A) were present in $58 \%$ and $42 \%$ of the cases, respectively. High score was associated with shorter median progression-free survival (PFS) and overall survival (OS) in comparison to low score (PFS: 11.4 vs. 25.46 months, $P=0.0005$; OS: 32.1 vs. $>90$ months, $P=0.0001$, respectively) (Figure $1 \mathrm{~B}$ and $\mathrm{C})$. High CD47 expression was associated with $17 \mathrm{p}$ (p53) deletions $(P=0.0407)$ and elevated $\beta$ 2 microglobulin level $(P=0.0323)$ (Online Supplementary Table S1), two well-known poor risk factors in $\mathrm{MM}$; it was also correlated with higher percentage of myeloma cells in the BM specimens $(P=0.0157)$ (Online Supplementary Figure $S 1 B$ ). Multivariate analysis adjusting above three variates confirmed that high CD47 expression was an independent poor risk factor for PFS ( $\mathrm{HR}=0.465$, $95 \%$ confidence 0 interval [CI]: 0.258-0.839, $P=0.0110$ ) and OS (HR=0.149, 95\%CI: $0.060-0.373, P<0.0001)$. There was no significant association between CD47 protein expression and other clinical or biological factors such as age, sex, hemoglobin, creatinine, calcium, albumin, and other cytogenetic risk factors including $13 \mathrm{q}$ deletion, $\mathrm{t}(4 ; 14), 1 \mathrm{p} 21$ deletion, and 1q21 (CKS1B) amplification (Online Supplementary Table S1). Moreover, in support of our experimental finding, analyses of publicly available data in the CoMMpass database for $767 \mathrm{MM}$ patients showed that OS and PFS were significantly shorter in patients with high CD47 mRNA level $(\mathrm{n}=383$ ) in comparison to patients with low CD47 mRNA level $(n=384)$ (Online Supplementary Figure S1C). In addition, the CD47 mRNA level was significantly higher in the MM cells from relapsed patients compared to newly diagnosed MM patients and normal plasma cells (Figure 1D). We have also identified correlation between higher CD47 expression level and progression of the disease in $\mathrm{MM}$ patients in bortezomib clinical trials (Online Supplementary Figure $S 1 D)$. In addition, we evaluated the endogenous CD47 expression in two drug-resistant MM cell lines (8226-R5 and MM.1R) and their parental lines (8226 and MM.1S). By Western blot the CD47 expression level was significantly higher in 8226-R5 and MM.1R cells in comparison to 8226 and MM.1S, respectively (Figure 1E), indicating that CD47 level is associated with drug response in MM cells.

\section{MiR-155 is down-regulated in multiple myeloma cells and directly targets CD47}

To elucidate a molecular mechanism underlying CD47 overexpression in drug resistant MM cells, several miRNA-target prediction algorithms were exploited $d^{19-21}$ to identify miRNAs which can potentially target CD47; 82 CD47 targeting miRNA candidates were found. Comprehensive bioinformatics analysis on miRNA/mRNA MM patient datasets on CD47 targeting miRNA candidates was carried out to identify the miRNAs that had a negative correlation with CD47 expression level in MM patients. We found four miRNAs (miR-425, miR-135b, and miR-326 and miR-155) were negatively correlated with CD47 expression (Figure 2A and Online Supplementary Figure S2A-C). Furthermore, analyzing the patient dataset revealed that among these 
A

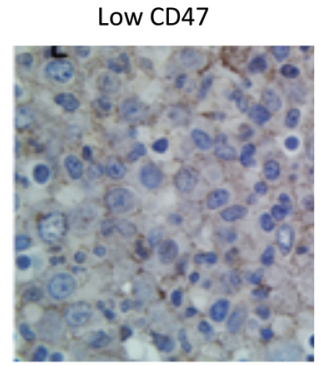

High CD47

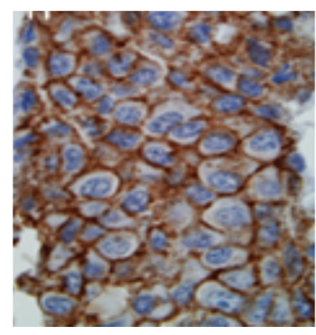

B

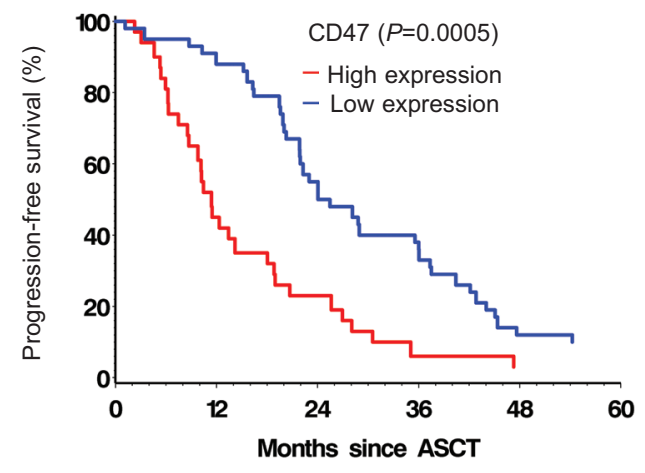

D

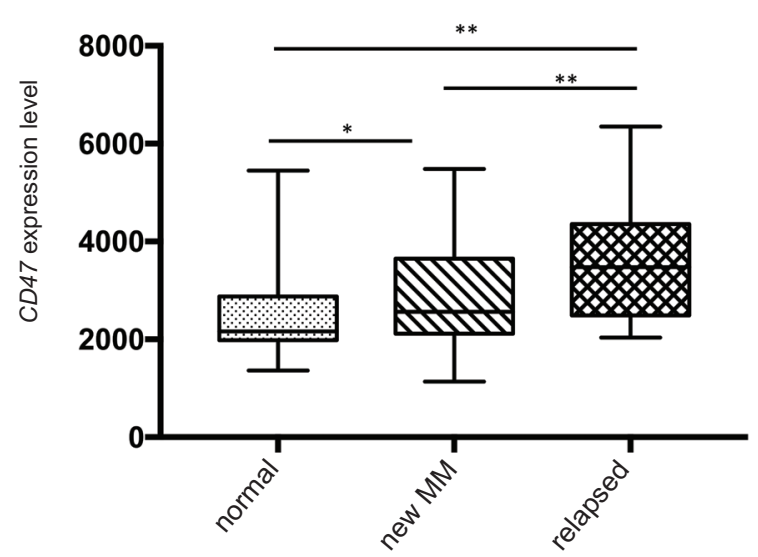

C

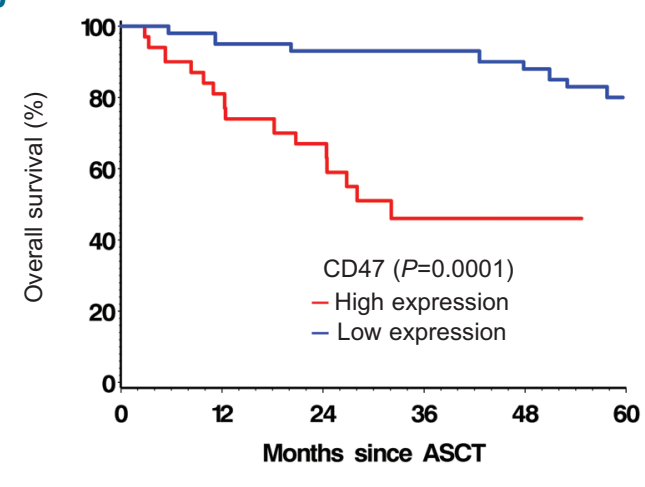

E

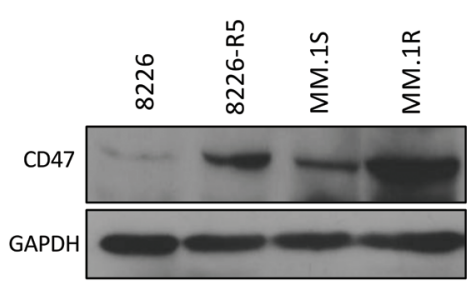

Figure 1. Upregulation of CD47 in multiple myeloma (MM) is correlated with poor patient survival. (A) Representative immunohistochemistry (IHC) staining with CD47 antibody in two MM patients with low and high expression of CD47. (B and C) Kaplan-Meier plots indicate the progression free survival (PFS) (B) and overall survival (OS) (C) for $67 \mathrm{MM}$ patients categorized by CD47 expression level. P-value is determined by log-rank test. (D) The box whisker plot shows CD47 expression in different stages of MM as shown in the graph, GSE6477 dataset. Normal donor (ND) $n=15$, newly diagnosed MM $n=73$, and Relapsed MM $n=28$. One-way analysis of variance and Student $t$-test was used. $P<0.05, * * P<0.01$ were considered as significant at $95 \%$ confidence interval. (E) CD47 expression was determined by Western blot analysis in 8226 versus 8226-R5 and MM.1S versus MM.1R cells, respectively. ASCT: autologous stem cell transplant.

miRNAs, miR-155 was significantly lower in stage III of the MM disease in comparison to stage I and II and healthy donor samples (Figure 2B), confirming the clinical relevance between miR-155 downregulation and the advanced stage of $\mathrm{MM}$ disease. In addition, the expression level of miR-155 was assessed in 8226-R5 and MM.1R resistant cell lines by quantitative PCR and compared to 8226 and MM.1S parental cells. MiR-155 showed a significant downregulation in 8226-R5 and MM.1R relative to 8226 and MM.1S, respectively (Figure 2C). To determine whether CD47 expression was selectively regulated by miR-155, we transfected two drug resistant MM cell lines with synthetic miR-155 mimics and identified that CD47 protein level was suppressed in
miR-155 transfected cell lines, suggesting miR-155 is a specific regulator of CD47 in myeloma cells (Figure 2D and E). The target scan analysis revealed a conserved domain within the $3^{\prime}$ UTR of CD47 with a potential miR155 binding site (Figure 2F). To validate CD47 as a direct target of miRNA-155, the 3'UTR sequence of human CD47 was cloned into the luciferase-expressing vector pEZX-MT01 downstream of the firefly luciferase gene. In order to further substantiate the site-specific repression of miR-155 on CD47, we constructed a mutant 3'UTR reporter clone and miR-155 binding site in which the 3'UTR of CD47 was inactivated by several mutations. Co-transfection of cells with the mutant or wild type of CD47-UTR luciferase reporter vectors together with miR- 
A

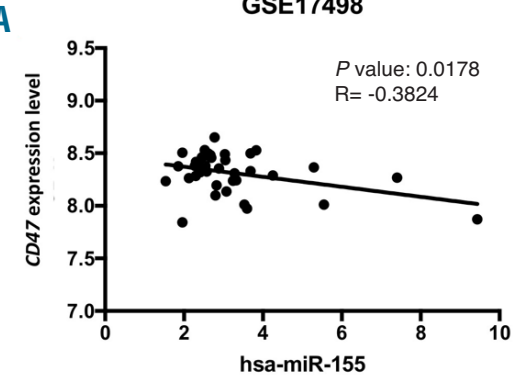

D

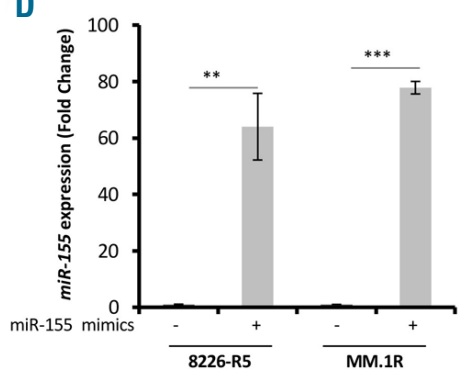

F
B

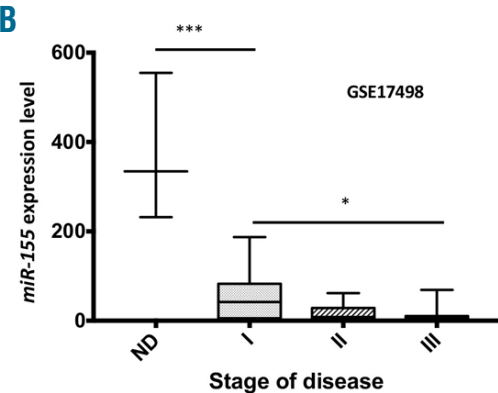

C

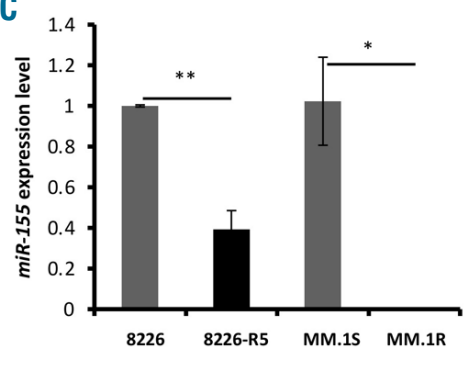

E
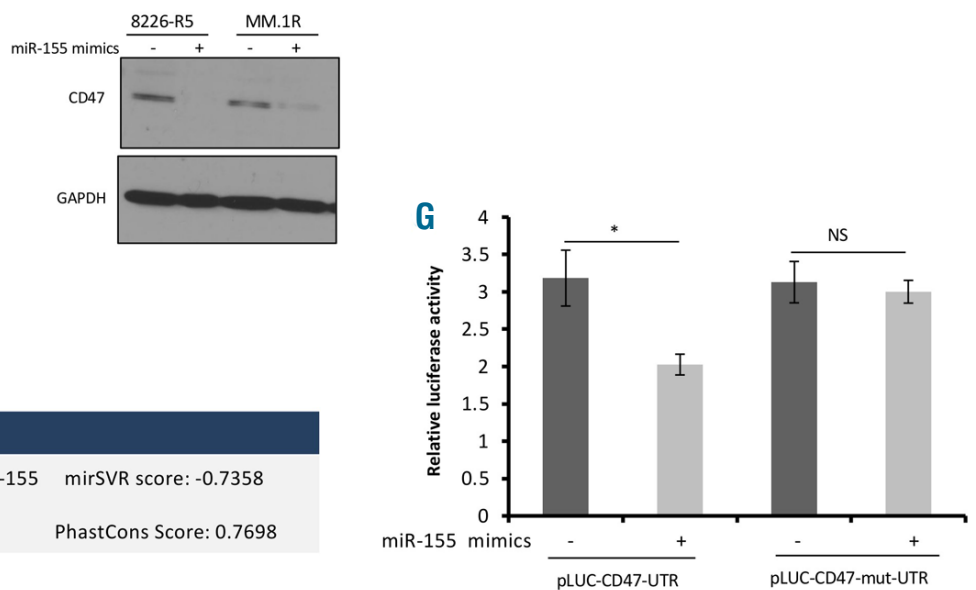

Figure 2. CD47 is a direct target of miR-155 in multiple myeloma (MM) cells. (A) Correlation analysis of endogenous miR-155 with CD47 expression in patient dataset (GSE17498, $\mathrm{n}=38 \mathrm{MM}$ patients) presented as scatter plots. Linear regression with Pearson's correlation coefficients ( $r$ ) and $P$-value were presented in the graph. (B) Box whisker plot showing miR-155 expression in GSE17498 dataset. Normal donor (ND): $n=3$, stage I (Durie-Salmon): $n=9$, stage II (Durie-Salmon): $n=9$ and stage III (Durie-Salmon): $n=16$. (C) The total RNA including miRNA was isolated from MM resistant and sensitive cell lines and the level of miR-155 was assessed by qualitative polymerase chain reaction (qPCR). The values were normalized to SNORD72. (D) The 8226-R5 and MM.1R cells were transfected with $20 \mu \mathrm{M}$ of either miR-155 mimics or scramble control using Hiperfect transfection reagent for 48 hours (h), and the level of miR-155 was assessed by qPCR. (E) The $8226-R 5$ and MM.1R cells were transfected with $20 \mu \mathrm{M}$ of either miR-155 mimics or scramble control using Hiperfect transfection reagent for $48 \mathrm{~h}$, and the whole cell lysate was subjected to Western blot with indicated antibodies. (F) The miR-155 binding site in the CD47 $3^{\prime}$-UTR. Putative conserved target sites in the CD47 3'-UTR were identified using the TargetScan algorithm. Matched nucleic acid-base pairs were linked as “-” (G) The 3' UTR sequence of human CD47 was cloned into the luciferaseexpressing vector pEZX-MT01 to the downstream of the firefly luciferase gene. For construction a mutant 3'UTR reporter clone, the miR-155 binding site in the 3'UTR of CD47 was inactivated by several mutations. The cells were transiently co-transfected with reporter plasmids (pEZX-MT-Control, pEZX-wt-CD47-3'UTR or pEZX-mutCD47-3'UTR) and miR-155 mimics or control miRNA. Cells were harvested $48 \mathrm{~h}$ after transfection and luciferase activities were analyzed as the relative activity of firefly to Renilla. Readings from the empty plasmid ( $\mathrm{pEZX-MT-control)} \mathrm{were} \mathrm{used} \mathrm{for} \mathrm{normalization.} * P<0.05, * * P<0.01, * * * P<0.001$ were considered as significant at $95 \%$ confidence interval. NS: not significant.

155 mimics or scramble control showed that miR-155 mimics significantly reduced wild type CD47-UTR reporter luciferase activity, but not that of the mutantUTR reporter (Figure 2G), indicating that miR-155 can directly target the CD47 3' UTR.

\section{MiR-155-mediated loss of cell surface CD47 promotes the phagocytosis of multiple myeloma cells}

Previous studies suggested that cells may lose surface CD47 during apoptosis to enable phagocytic clearance. ${ }^{22,23}$ To demonstrate the effect of miR-155 on cell surface CD47 level, we transfected two drug resistant MM cell lines with synthetic miR-155 mimics. The FACS analysis revealed that the level of cell surface CD47 was significantly decreased in miR-155 over-expressing resistant MM cells (Figure 3A). Blocking CD47 with anti-CD47 monoclonal antibodies has enabled the phagocytosis of myeloma cells. ${ }^{24}$ We investigated whether targeting of CD47 by miR-155 overexpression promotes the phagocytosis of myeloma cells. Our results showed that macrophage-like cells differentiated from THP-1 mono- cytes phagocytosed human myeloma cells at a low frequency when treated with scramble control. However, miR-155 overexpression in drug resistant 8226-R5 and MM.1R MM cells significantly increased the phagocytosis of the cells (Figure $3 \mathrm{~B}$ and C). Additionally, we explored whether overexpression of CD47 can rescue the effect of miR-155 on phagocytosis of myeloma cells by macrophages. To this end, we performed functional rescue assays by the ectopic expression of CD47 in 8226-R5 and MM.1R resistant cells and co-transfecting with synthetic miR-155 mimics or miRNA negative control. The CD47 overexpression partially abolished the phagocytosis of $\mathrm{MM}$ cells by macrophages confirming that targeting of CD47 by miR-155 increased the phagocytosis of resistant MM cells (Figure 3D and E). These data indicate that increased phagocytosis of myeloma cells by high level miR-155 expression results as a consequence of CD 47 targeting by miR-155 which leads to reduction of cell surface CD47 on MM cells and consequently reduction of the 'do not eat me signal', followed by phagocytosis of MM cells by macrophages. 
A
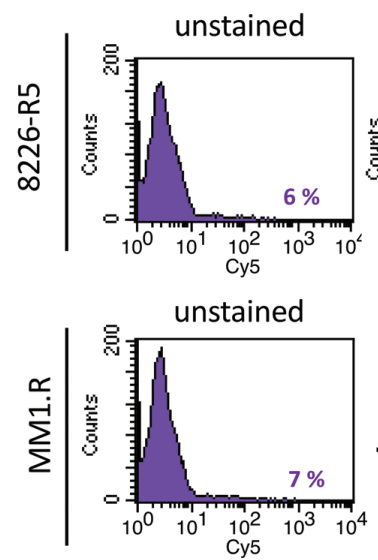

B

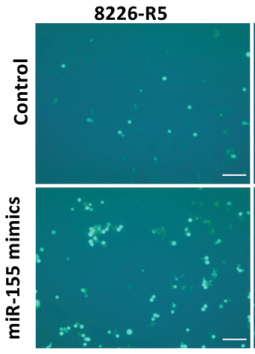

D
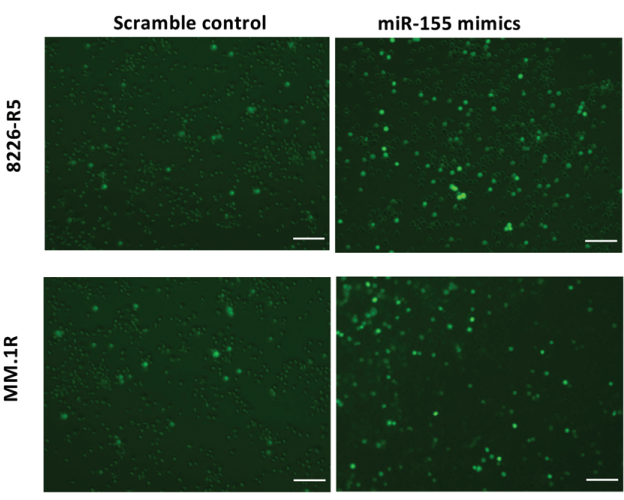

Transduced with empty vector

E

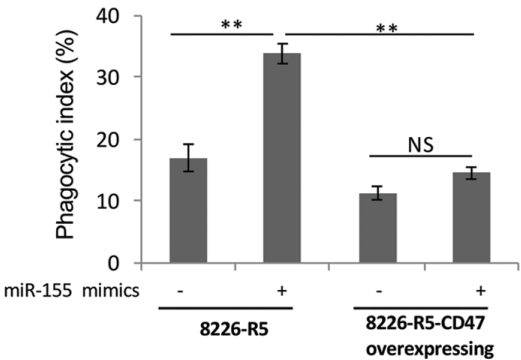

miR-155 mimics

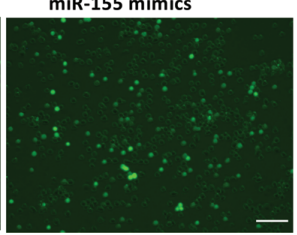

miR-155 mimics
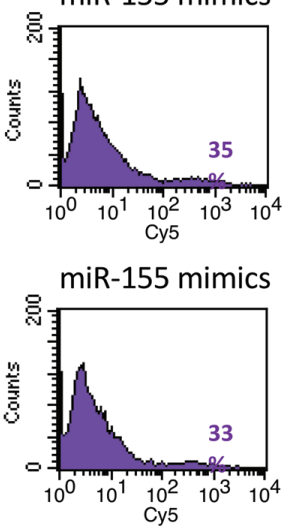

C

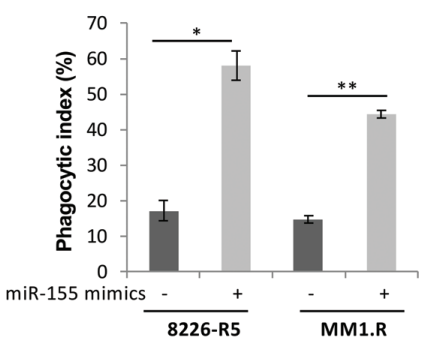

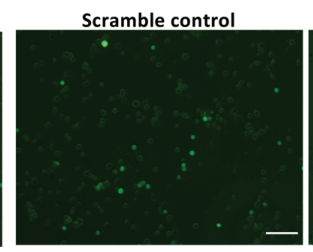
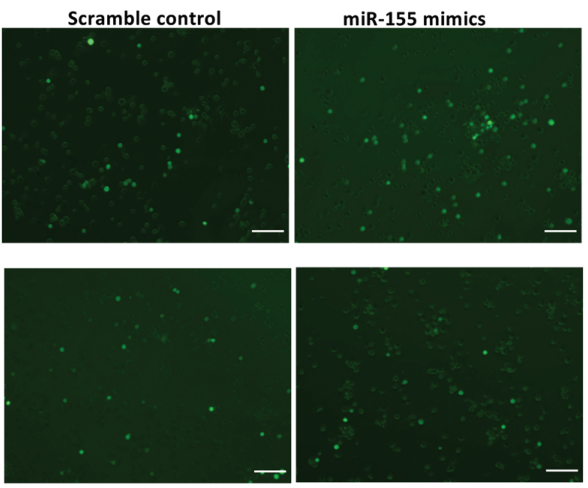

Transduced with CD47 vector

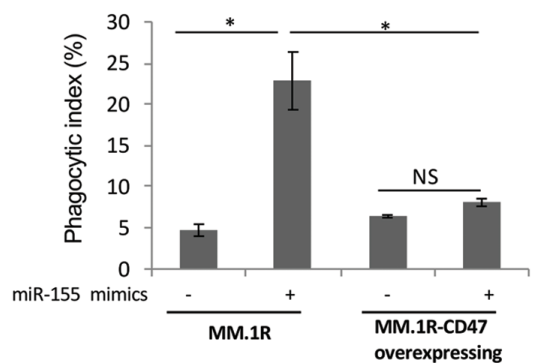

Figure 3. Targeting of CD47 by miR-155 increased the phagocytosis of myeloma resistant cells. (A) The 8226-R5 and MM.1R cell lines were transfected with synthetic miR-155 or scramble control for 48 hours (h) and stained with CD47 antibody and analyzed by flow cytometry to determine the level of CD47 in the cell surface of multiple myeloma (MM) cells. The percentage of CD47 stained cells is indicated in each histogram. (B) The GFP-tagged 8226-R5 and MM.1R cell lines were transfected with synthetic miR-155 or scramble control for $48 \mathrm{~h}$ and co-cultured with THP-1 like macrophages for $2 \mathrm{~h}$ at $37^{\circ}$. Macrophages were repeatedly washed four times with PBS $1 \mathrm{X}$ and subsequently imaged with fluorescent microscope. A representative result of each condition is shown. Scale bars are 100 um. (C) The phagocytic index was calculated as the number of phagocytosed GFP+ with CD47 lentiviral or empty vector. The CD47 transduced or control cells were transfected with synthetic miR-155 or scramble control for $48 \mathrm{~h}$ and co-cultured with THP-1 like macrophages for $2 \mathrm{~h}$ at $37^{\circ}$. Macrophages were repeatedly washed and subsequently imaged with fluorescent microscope. A representative result of each condition is shown. Scale bars are $100 \mu \mathrm{m}$. (E) The phagocytic index was calculated as the number of phagocytosed GFP ${ }^{+}$cells per 100 macrophages. $\star P<0.05$, $\star \star P<0.01$, were considered as significant based on the $95 \%$ of confidence intervals. The result is the sum of three triplicate experiments. NS: not significant. 
A
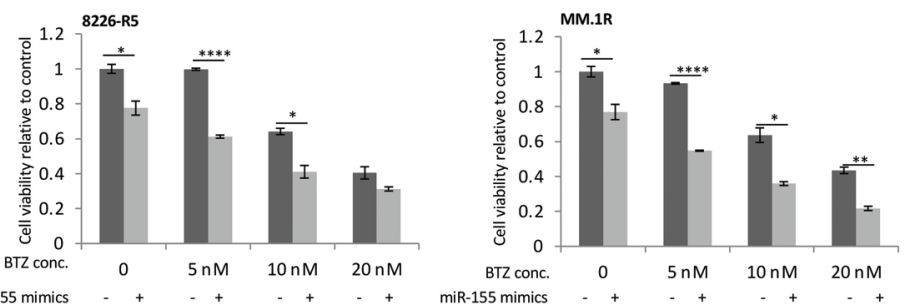

miR-155 mimics $-+\quad+++++$ miR-155 mimics
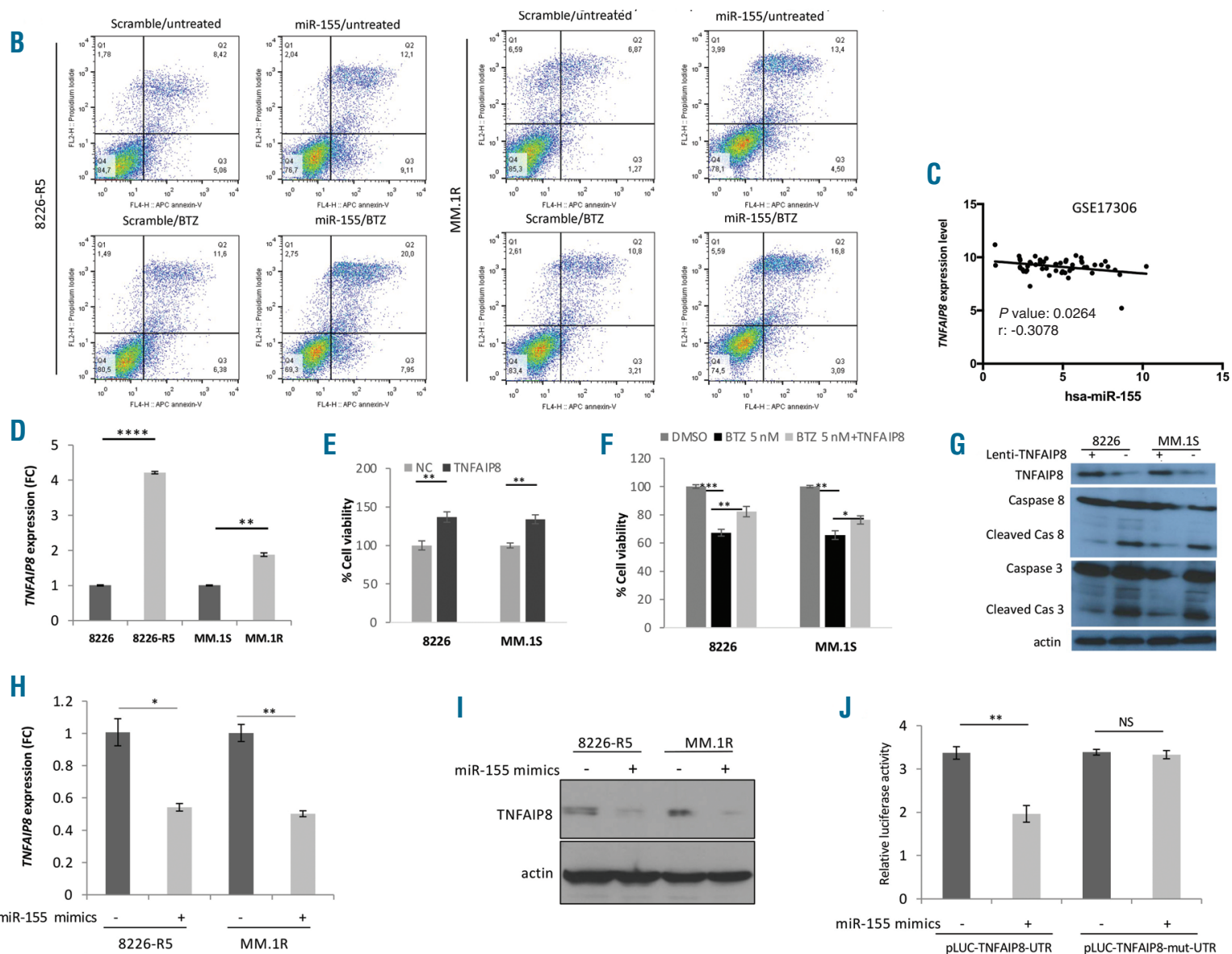

G
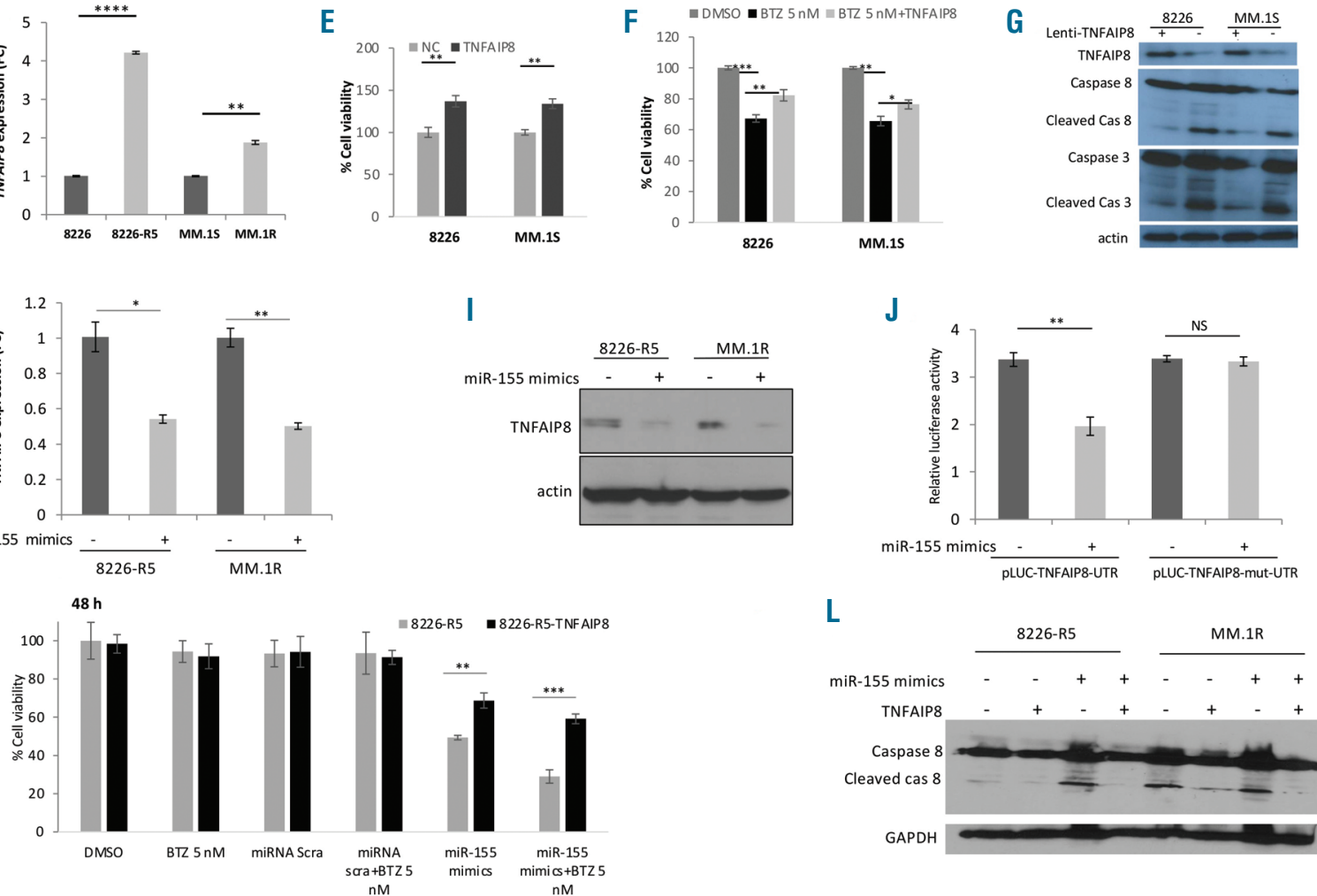

$\mathbf{L}$

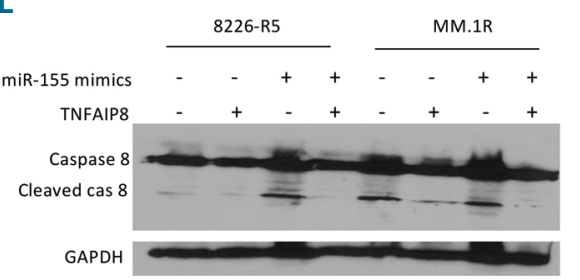

Figure 4. miR-155 restoration inhibits cell proliferation and induces apoptosis in multiple myeloma (MM) cells. (A) The 8226-R5 and MM.1R cell lines were trans fected with synthetic miR-155 or scramble control and treated with different concentrations of BTZ for 48 hours (h) and cell viability was measured using MTT assay. The result is the sum of three triplicate experiments. (B) The 8226-R5 and MM.1R cells were transfected with $20 \mu \mathrm{M}$ of either miR-155 mimics or scramble control using Hiperfect transfection reagent for $24 \mathrm{~h}$, and then treated with $10 \mathrm{nM}$ BTZ or vehicle for $24 \mathrm{~h}$. Then the cells were stained with annexin-V/propidium iodide and analyzed by flow cytometry to determine the percentage of apoptotic cells. The percentage of double positive cells indicated in each dot plot as a representation of apoptosis. (C) Correlation analysis of endogenous miR-155 with TNFAIP8 expression in patient dataset (GSE17306) presented as scatter plots. Linear regression with Pearson's correlation coefficients $(r)$ and $P$-value were presented in the graph. (D) TNFAIP8 expression was determined by qualitative-polymerase chain reaction (q-PCR) a in 8226 versus 8226-R5 and MM.1S versus MM.1R cells, respectively. (E and F) Cell proliferation was assessed in RPMI-8226 and MM.1S cells with or without stable TNFAIP8 overexpression alone or in combination with $5 \mathrm{nM}$ BTZ. (G) RPMI-8226 and MM.1S parental cell and TNFAIP8 over-expressing cells were subjected to Western blot with indicated antibodies. ( $\mathrm{H}$ and I) The 8226-R5 and MM.1R cells were transfected with $20 \mu \mathrm{M}$ of either miR-155 mimics or scramble control using Hiperfect transfection reagent for $48 \mathrm{~h}$, and the TNFAIP8 level was assessed with q-PCR and Western blot. (J) The cells were transiently co-transfected with reporter plasmids (pEZX-MT-Control, pEZX-wt-TNFAIP8-3'UTR or pEZX-mut- TNFAIP8-3'UTR) and miR-155 mimics or control miRNA. Cells were harvested 48 h after transfection and luciferase activities were analyzed as the relative activity of firefly to Renilla. Readings from the empty plasmid ( $p E Z X-M T-c o n t r o l)$ were used for normalization. (K) The 8226-R5 cells with or without stable TNFAIP8 overexpression were transfected with synthetic miR-155 or scramble control. Cell proliferation was assessed in the absence or presence of $5 \mathrm{nM} \mathrm{BTZ} 48 \mathrm{hrs}$ after treatment (L). The 8226-R5 cells with or without stable TNFAIP8 overexpression were transfected with synthetic miR-155 or scramble control. The cell lysate was prepared $48 \mathrm{~h}$ after transfection and subjected to western blot with indicated antibodies. $* P<0.05$, $* * P<0.01, * * * P<0.001, * * * * P<0.0001$ and significant difference based on the $95 \%$ of confidence intervals. NS: not significant. 
MiR-155 inhibits multiple myeloma cell proliferation and induces apoptosis in resistant myeloma cells by targeting TNFAIP8

To examine the functional effect of miR-155 on cell proliferation and apoptosis in drug resistant MM cells, two resistant cell lines (8226-R5 and MM.1R) were transfected with miR-155 mimics or scramble control. We found that transfection of synthetic miR-155 in drug resistant cells could re-sensitize the resistant cells to BTZ in a dose and time dependent manner (Figure 4A and Online Supplementary Figure S3A). Furthermore, reduction of cell proliferation upon overexpression of miR-155 was associated with apoptosis of the resistant cells as indicated by an increase in the percentage of annexin- $V$ positive cells compared to the control (Figure 4B). Collectively, these results support the concept that miR-155 functions as a tumor suppressor miRNA and its dysregulation contributes to MM cell growth and drug resistance. To understand the molecular mechanism of action of miR-155 on apoptosis, several MicroRNA target prediction databases were analyzed and the targets with highest target scores in at least four different databases were selected. Moreover, we assessed the oncogenic potential of the selected genes using OncoScore Internet-based tool (Online Supplementary Figure S3B). Furthermore, analyzing the MM patient datasets on the targets with the most oncogenic scores revealed that among these targets, TNFAIP8, a negative mediator of apoptosis, showed more clinical relevance with MM disease. Target scan analysis showed miR-155 can potentially target TNFAIP8 3'UTR (Online Supplementary Figure S3C). We identified a negative correlation (with the low/moderate coefficient value -0.24 , -0.26 and -0.3$)$ between miR-155 and TNFAIP8 mRNA expression level in MM patient samples in comparison to other potential targets (Figure 4C and Online Supplementary Figure S3D and E). We also found that the mRNA expression level of TNFAIP8 was higher in MM patients at relapsed stage compared to those at diagnosis (Online Supplementary Figure S3F). Moreover, in support of our finding, we evaluated the TNFAIP8 expression in two drugresistant MM cell lines (8226-R5 and MM.1R) and their parental lines (8226 and MM.1S). We found that the TNFAIP8 $\mathrm{mRNA}$ and protein levels were significantly higher in 8226-R5 and MM.1R cells in comparison to 8226 and MM.1S, respectively (Figure 4D and Online Supplementary Figure S3G). Notably, ectopic expression of TNFAIP8 in the parental cell lines promoted cell proliferation and increased resistance to BTZ by inhibiting caspase-8 activity and subsequently caspase-3 activation (Figure 4E-G), indicating that TNFAIP8 can modulate drug response in MM cells. In addition, overexpression of miR-155 in drug resistant MM cells significantly reduced mRNA and protein level of TNFAIP8 (Figure $4 \mathrm{H}$ and $\mathrm{I}$ ).

To validate TNFAIP8 as a direct target of miR-155, the 3' UTR of human TNFAIP8 was cloned into the luciferaseexpressing vector pEZX-MT01 downstream of the firefly luciferase gene. We also constructed a mutant 3'UTR reporter clone. To this end, the miR-155 binding site in the $3^{\prime}$ UTR of TNFAIP8 was inactivated by several insertions in miRNA binding site. Co-transfection of cells with the mutant or wild type of TNFAIP8-UTR luciferase reporter vectors together with miR-155 mimics or scramble control showed that miR-155 mimics significantly reduced wild type TNFAIP8-UTR reporter luciferase activity, but not that of the mutant-UTR reporter (Figure 4J), indicating that miR-
155 can directly target the TNFAIP8 $3^{\prime}$ UTR. Reversely, overexpression of TNFAIP8 in MM.1S cells could significantly decrease the miR-155 expression level, indicating that miR-155 is also regulated by TNFAIP8 through a negative feedback loop (Online Supplementary Figure S3H). Additionally, we explored whether TNFAIP8 can rescue the effect of miR-155 on MM cell growth. To this end, we performed functional rescue assay by over-expressing TNFAIP8 in 8226-R5 and MM.1R resistant cells and cotransfecting with synthetic miR-155 mimics or miRNA scramble control. The MTT results revealed that TNFAIP8 overexpression partially abolished the anti-tumor effect induced by miR-155 plus BTZ treatment confirming that combining miR-155 and BTZ induces a growth inhibitory effect in MM cells by targeting TNFAIP8 (Figure $4 \mathrm{~K}$ and Online Supplementary Figure S3I). In addition, Western blot results also confirmed that TNFAIP8 suppressed the TNFmediated apoptosis in MM resistant cells by inhibiting caspase-8 activity, subsequently resulting in inhibition of BID cleavage and caspase-3 activation (Figure 4L). Collectively, these results support the concept that miR-155 functions as a tumor suppressor miRNA and contributes to the effect of CD47 on MM cell growth and drug resistance.

\section{Restoration of miR-155 by miRNA mimics suppresses tumorigenesis in multiple myeloma xenograft model}

To translate our findings to a therapeutic model, we next investigated the effect of miR-155 overexpression on tumorigenesis of $\mathrm{MM}$ resistant cells in vivo. We established a mouse xenograft model with 8226-R5 resistant MM cells in severe combined immunodeficient (SCID) mice and treated them with miR-155 mimics alone or in combination with BTZ. Intratumoral injection of miR-155 significantly suppressed tumorigenesis in combination with BTZ (Figure 5A) without showing any untoward toxicity as indicated by the body weight (Figure 5B). The combination treatment of miR-155 mimics and BTZ significantly extended the OS in comparison to all other three treatments (Figure 5C). To assess in vivo targeting of TNFAIP8 by miR-155, we evaluated the level of TNFAIP8 and CD47 in mice tumors. Consistent with our in vitro data, the protein levels of TNFAIP8 were dramatically decreased in miR-155-treated groups compared with control (Figure 5D). We also verified the efficiency of miR-155 mimics delivery into tumor cells by quantitative (q)-PCR (Figure 5E). IHC analysis of tumor sections showed that treatment with miR-155 mimics and BTZ resulted in a decrease in the proliferation index (Ki67) and an increase in the apoptotic index (Tunnel), compared to either BTZ or miR-155 mimics alone (Figure 5F). These findings indicate that targeting of TNFAIP8 by miR- 155 sensitizes myeloma cells to BTZ treatment and contributes to the marked induction of apoptosis of MM cells, as well as suppression of MM tumor growth in vivo. The proposed model for mechanism of MM drug resistance is shown in Figure 6.

\section{Discussion}

Increased CD47 expression has been reported in various patient tumor cells and in some cases, CD47 high level expression was correlated with a worse prognosis. ${ }^{5,6,25}$ Examination of global gene expression profiling of $\mathrm{MM}$ patients revealed that CD47 expression is up-regulated in MM patients in comparison to MGUS and normal donor 
plasma cell samples. ${ }^{26}$ In addition; flow cytometric results demonstrated that CD47 protein expression was higher on MM cells in comparison to non-myeloma cells. ${ }^{24}$ To validate clinical relevance of CD47 overexpression on patient's outcome, several public datasets for MM patients were analyzed and revealed that CD47 expression was significantly higher in relapsed patients in comparison to newly diagnosed $\mathrm{MM}$ patients. Moreover, we performed
IHC in a cohort of MM patients and to the best of our knowledge, this is the first study to demonstrate that high CD47 protein expression was associated with unfavorable patient's outcome. Therefore, therapeutic intervention with CD47 targeting could be used as a promising strategy to treat MM. Consistent with our results, a recent IHC study on the clinical specimens of gastric cancer also indicated that CD47 positivity was an independent adverse
A

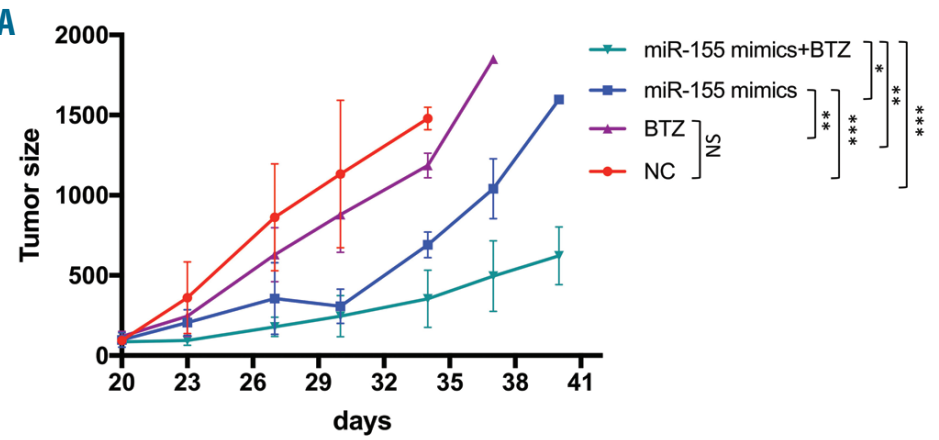

C

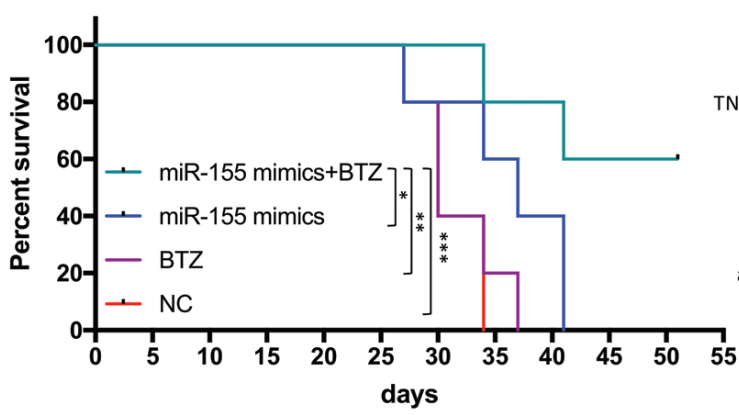

$\mathbf{F}$

control

$H \& E$

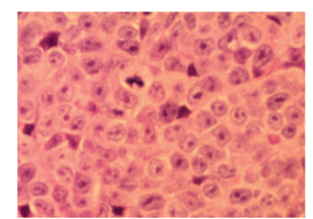

Tunel

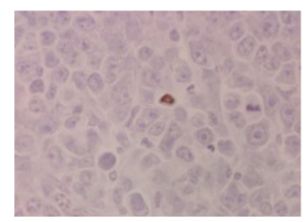

Ki67

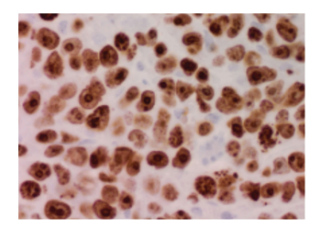

D

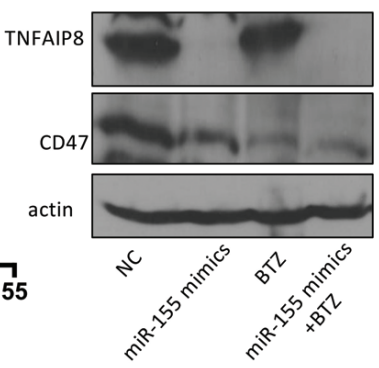

BTZ
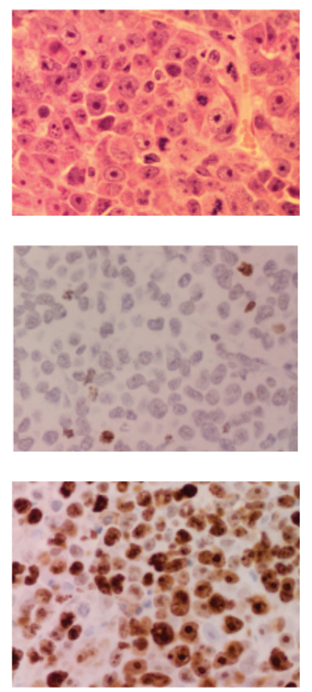

miR-155 mimics
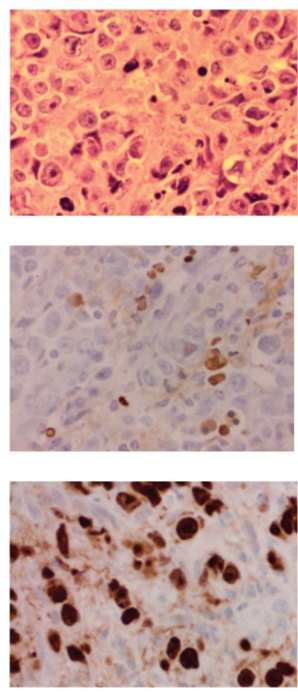

B

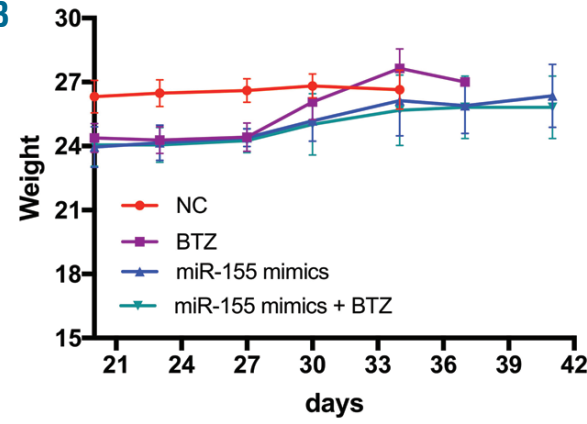

E

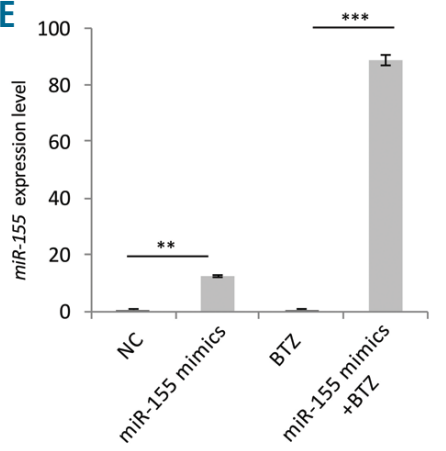

BTZ+miR-155 mimics
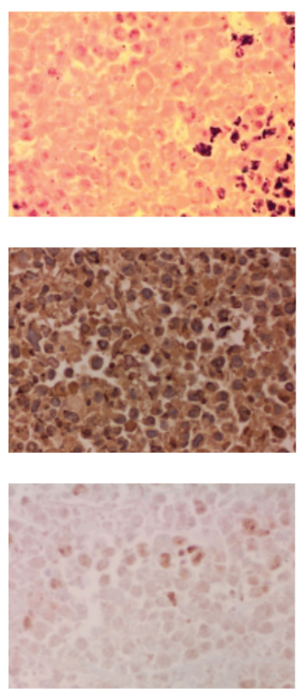

Figure 5. Synthetic miR-155 retards tumor growth and prolongs survival in human multiple myeloma (MM) mouse xenograft model. Intratumoral injection with either miR-155 mimics or control miRNA $(1 \mathrm{mg} / \mathrm{kg}$ ), with or without intraperitoneal injection of $0.5 \mathrm{mg} / \mathrm{kg}$ BTZ ( $\mathrm{n}=5 \mathrm{mice}$ per group) was carried out at an interval of 3 days for 15 days. (A) Overexpression of miR-155 enhanced BTZ-induced retardation of tumor growth in vivo. (B) Body weight was measured from first day of drug injection (day 20) every 3 days till day 42 and presented as mean \pm standard error mean (SEM). (C) Survival was evaluated using Kaplan-Meier curves and log-rank analysis from the first day of tumor cells injection until death or occurrence of an event. (D) Mice tumors from in vivo experiment were analyzed by immunoblotting for CD47 and TNFAIP8 protein expression. (E) The total RNA including miRNA was isolated from the mice of four groups and the level of miR-155 was measured by qualitative-polymerase chain reaction (q-PCR) to evaluate the delivery efficiency of miRNA mimics into tumor cells after intratumoral injection of miR-155 mimics using the novel formulation of neutral lipid emulsion (NLE; MaxSuppressor in vivo RNA Lancer II, BIOO Scientific). Fold change was expressed as log2-fold induction over control group (mean \pm SEM). (F) Representative microscopic images of immunohistochemical analysis of tumor sections from four treated groups with hematoxylin \& eosin, the proliferation index (Ki-67) and the apoptotic index, TUNEL staining. $* P<0.05, * * P<0.01$, $* * * P<0.001$, and significant difference based on the $95 \%$ of confidence intervals. 
prognostic factor. ${ }^{27}$ Our finding also showed that high CD47 protein expression was associated with higher BM myeloma cell infiltration. A possible explanation is that high CD47 expression could cause the escape of MM cells from immune surveillance and result in progression of the disease.

To understand the mechanism of increased expression of CD47, we found an inverse correlation between the expression of CD47 and its potential negative regulator, miR-155. We provided strong evidence that miR-155 negatively regulated CD47 expression in MM. miR-155 was down-regulated in drug resistant $\mathrm{MM}$ cell lines compared to parental lines and inversely correlated with the progression of MM disease. We also showed that there was a significant negative correlation between miR-155 and CD47 expression levels in $\mathrm{MM}$ patients. The luciferase assay also revealed that 3'UTR of CD47 was directly targeted by miR-155. In line with these findings, miRNA related CD47 overexpression has been reported in solid tumors, with high expression of CD47 being correlated with poor clinical outcome and tumor progression. ${ }^{28}$ Moreover, considering our observation that miR-155 mimics can downregulate CD47 protein in MM cells, and CD47 is reportedly a direct target of miR-155 in other contexts such as multiple sclerosis and adipogenesis, ${ }^{29,30}$ it indicates that low level of miR-155 in myeloma cells may contribute to upregulation of CD47. Furthermore, an important finding here is that overexpression of miR-155 in drug resistant MM cells could decrease the CD47 on the cell surface and induce phagocytosis by macrophages through activation of an 'eat me' signal in resistant cells, whereas overexpression of CD47 antagonized this effect of miR-155. Consistent with our results, another study demonstrated that blocking CD47 increased phagocytosis of myeloma cells (in vitro) induced tumor regression and alleviated bone resorption in animal models. ${ }^{31}$

We demonstrated that restoration of miR-155 level in drug resistant MM cells by miRNA mimics could efficiently reduce cell proliferation and induce apoptosis in resistant cells by targeting of TNFAIP8. It should be mentioned that TNFAIP8 is known to counteract apoptosis by inhibiting caspase-8 activity, subsequently resulting in inhibition of BID cleavage and caspase- 3 activation. ${ }^{32,33}$ It has also been shown that TNFAIP8 might serve as a predominant pro-tumor factor by modulating different modulators and molecular targets such as growth factor receptors (EGFR and VEGFR), cell cycle protein (Cyclin D1, phospho-Rb), cell surface transmembrane receptor (Integrin).${ }^{34}$ We found that TNFAIP8 was significantly upregulated in drug resistant $\mathrm{MM}$ cells compared to parental cells. Analysis of the MM patient dataset also revealed that it was elevated in MM patients at relapsed stage in comparison to presentation time point, suggesting that TNFAIP8 is an oncogene and could contribute to drug resistance in $\mathrm{MM}$. Along this line, other studies found evidence linking TNFAIP8 oncogene to the survival of several cancer types. ${ }^{35,36}$ Moreover, we have demonstrated that TNFAIP8 overexpression increased cell proliferation of

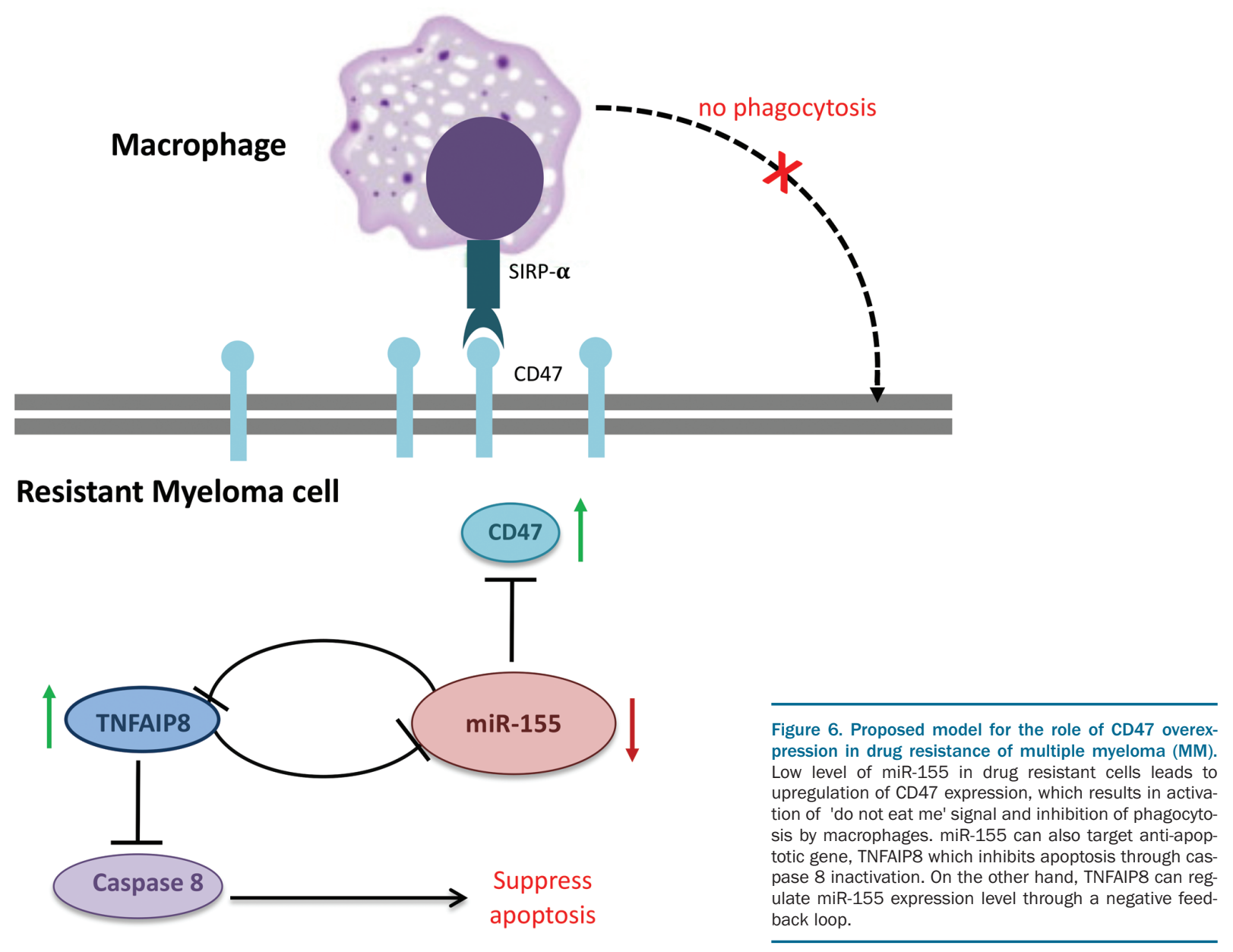


parental sensitive $\mathrm{MM}$ cells and conferred resistance to BTZ in parental MM cells. Of note, it has also been shown that TNFAIP8 overexpression was correlated with drug resistance in several cancers including $\mathrm{MM}^{37-39}$ Collectively, these data suggest that TNFAIP8 has a significant role in the oncogenesis and progression of human malignancies. Interestingly, we demonstrated that targeting of TNFAIP8 by miR-155 could re-sensitize the drug resistant $\mathrm{MM}$ cells to anti-myeloma drugs, and TNFAIP8 overexpression partially rescued the cytotoxic effect of miR-155 in drug resistant MM cells. These results suggest that, besides directly targeting the cell surface protein CD47 and promoting phagocytosis, ectopic expression of miR-155 could target the oncogene TNFAIP8 and induce apoptosis at the same time (Figure 6), whereas the previous studies found that anti-CD47 therapy could just increase phagocytosis of MM cells but did not induce antibody-dependent cell-mediated cytotoxicity (ADCC) or complement-dependent cytotoxicity (CDC) on myeloma cells. $^{24}$

Our pre-clinical study on xenograft MM mouse model illustrated that synthetic miR-155 mimics can restrain the tumor growth and prolong survival of the resistant MM mice model. In line with these findings, a very recent study also showed that miR-155 has tumor suppressor activity in MM cells and antagonized bortezamib resistanse by targeting proteasome subunit $\beta 5 .^{40}$ Several studies have demonstrated that miRNA may be applied for the targeted delivery of personalized medicine to improve the outcome of MM patients and the number of studies focusing on pre-clinical applications of miRNAs in $\mathrm{MM}$ is increasing. ${ }^{41-44}$ Importantly, formulated NLE-miR-34a was safely administered to mice bearing MM tumors by intratumor injection, suggesting a favorable therapeutic index for synthetic miRNAs. ${ }^{45}$ In particular, miRNA-based therapeutics can be relevant both for safety issues and to abrogate late onset of resistance because of the complexity of miRNA-targeted pathways and the consequent low chance of developing individual 'escape' mutations in the treated cells. In addition, although current therapeutic methods such as small molecule inhibitors or monoclonal antibodies have shown promise, there are many genes that are not druggable using these methods. An advantage of miRNA-based therapy is the ability to rapidly develop new therapies with broad adaptability. In addition, miRNA offers the opportunity to target multiple mRNA targets with a single miRNA in a given pathway. ${ }^{46,47}$

It is important to mention that, although miR-based therapeutics have demonstrated great promise for the treatment of different diseases, this is still an evolving field. The main obstacle for clinical application of RNAbased therapeutics is determining how to best deliver the agent to targeted cells. Recent progress in miRNA delivery such as nanoparticle-based technology shows great promise as it may reduce doses, which will be beneficial for treatment of cancer. ${ }^{48}$ Other factors such as safety, efficacy, and target selection will also require optimization to produce successful drugs. Engineered nanoparticles are especially used for delivery to specific cells, which will help to achieve this goal. RNA-based therapeutics combined with conventional chemotherapy agents might be a new approach to use for cancer treatment and will ultimately help to bring the RNA-based therapeutics strategy to the clinic. Nevertheless, therapeutic miRNAs definitely have the potential to contribute significantly to the future of medicine. ${ }^{42,49,50}$ In the current study, local administration of miRNA has been applied, which is the most commonly used model for miRNA-based therapeutic. Importantly, our study provides a proof of principle that miR-155 can effectively eradicate tumor growth in vivo by targeting CD47 and TNFIP8. In future studies, a xenograft model would be required to confirm the efficacy of the treatment, with an alternative route of administration.

In conclusion, we show that CD47 could serve as an adverse prognostic factor in $\mathrm{MM}$ and demonstrate a novel mechanism of miR-155/CD47/TNFAIP8 axis in MM drug resistance. We illustrate a tumor suppressor role for miR155 in MM, which contributes to deregulation of CD47 and TNFAIP8 oncogene. Therefore, targeting CD47 by miR-155 mimics implies a novel therapeutic strategy for relapsed/refractory $\mathrm{MM}$, particularly with high CD47 expression.

\section{Acknowledgments \\ We thank Drs. Mark Minden and Eldad Zacksenhaus for helpful discussions.}

\section{Funding}

The study was supported in part by the grants from Leukemia and Lymphoma Research Society of Canada (LLSC), and Cancer Research Society (CRS).

\section{References}

1. Rollig C, Knop S, Bornhauser M. Multiple myeloma. Lancet. 2015;385(9983):21972208.

2. Sonneveld P and Broij A. Treatment of relapsed and refractory multiple myeloma. Haematologica. 2016;101(4):396-406.

3. Barclay AN, Berg TK. The interaction between signal regulatory protein alpha (SIRP $\alpha$ ) and CD47: structure, function, and therapeutic target. Annu Rev Immunol. 2014;32:25-50.

4. Oldenborg PA, Gresham HD, Lindberg FP. CD47-signal regulatory protein alpha (SIRPalpha) regulates Fc gamma and complement receptor-mediated phagocytosis. J Exp Med. 2001;193(7):855-862.
5. Majeti R, Chao MP, Alizadeh AA, et al. CD47 is an adverse prognostic factor and therapeutic antibody target on human acute myeloid leukemia stem cells. Cell. 2009;138(2):286-299.

6. Chao MP, Alizadeh AA, Tang C, et al. AntiCD47 antibody synergizes with rituximab to promote phagocytosis and eradicate nonHodgkin lymphoma. Cell. 2010;142(5):699713.

7. Galli S, Zlobec I, Schürch C, et al. CD47 protein expression in acute myeloid leukemia: a tissue microarray-based analysis. Leuk Res. 2015;39(7):749-756.

8. Rendtlew Danielsen JM, Knudsen LM, Dahl IM, Lodahl M, Rasmussen T. Dysregulation of CD47 and the ligands thrombospondin 1 and 2 in multiple myeloma. Br J Haematol. 2007;138(6):756-760.
9. Muz B, Kusdono H D, King J, et. al. Targeting CD47 As a novel therapeutic strategy in multiple myeloma. Blood. 2017; 130(Suppl 1):3099.

10. Tucci M, Stucci S, Strippoli S, Dammacco F, Silvestris F. Dendritic cells and malignant plasma cells: an alliance in multiple myeloma tumor progression? Oncologist. 2011; 16(7):1040-1048.

11. Seckinger A, Meißner T, Moreaux J, et al miRNAs in multiple myeloma-a survival relevant complex regulator of gene expression. Oncotarget. 2015;6(36):39165-39183.

12. Zhang YK, Wang $\mathrm{H}$, Leng $\mathrm{Y}$, et al. Overexpression of microRNA-29b induces apoptosis of multiple myeloma cells through down regulating Mcl-1. Biochem Biophys Res Commun. 2011;414(1):233-239.

13. Kumar D, Whiteside TL, Kasid U 
Identification of a novel tumor necrosis factor-alpha-inducible gene, SCC-S2, containing the consensus sequence of a death effector domain of fas-associated death domainlike interleukin-1beta-converting enzymeinhibitory protein. J Biol Chem. 2000;275(4):2973-2978

14. You Z, Ouyang $\mathrm{H}$, Lopatin D, Polver PI, Wang CY. Nuclear factor-kappa B-inducible death effector domain-containing protein suppresses tumor necrosis factor-mediated apoptosis by inhibiting caspase-8 activity. J Biol Chem. 2001;276(28):26398-26404.

15. Kumar D, Gokhale P, Broustas C, Chakravarty D, Ahmad I, Kasid U. Expression of SCC-S2, an antiapoptotic molecule, correlates with enhanced proliferation and tumorigenicity of MDA-MB 435 cells. Oncogene. 2004;23(2):612-616.

16. Buzzeo R, Enkemann S, Nimmanapalli R, et al. Characterization of a R115777-resistant human multiple myeloma cell line with cross-resistance to PS-341. Clin Cancer Res. 2005;11(16):6057-6064

17. Yang Y, Chen Y, Saha MN, et al. Targeting phospho-MARCKS overcomes drug-resistance and induces antitumor activity in preclinical models of multiple myeloma. Leukemia. 2015;29(3):715-726.

18. Saha MN, Chen Y, Chen $M H$, Chen G, Chang H. Small molecule MIRA-1 induces in vitro and in vivo anti-myeloma activity and synergizes with current anti-myeloma agents. Br J Cancer. 2014;110(9):22242231.

19. Wang $X$. miRDB: a microRNA target prediction and functional annotation database with a wiki interface. RNA. 2008; 14(6):1012-1017.

20. Wang X, El Naqa IM. Prediction of both conserved and non-conserved microRNA targets in animals. Bioinformatics. 2008; 24(3):325-332.

21. Betel D, Wilson M, Gabow A, Marks DS, Sander C. The microRNA.org resource: targets and expression. Nucleic Acids Res. 2008;36(Database issue):D149-153.

22. Burger P, Hilarius-Stokman P, de Korte D, van den Berg TK, van Bruggen R. CD47 functions as a molecular switch for erythrocyte phagocytosis. Blood. 2012; 119(23):5512-5521

23. Khandelwal S, van Rooijen N Saxena RK. Reduced expression of $\mathrm{CD} 47$ during murine red blood cell (RBC) senescence and its role in $\mathrm{RBC}$ clearance from the circulation. Transfusion. 2007;47(9):1725-1732.

24. Kim D, Wang J, Willingham SB, Martin R, Wernig G, Weissman IL. Anti-CD47 antibodies promote phagocytosis and inhibit the growth of human myeloma cells.
Leukemia. 2012;26(12):2538-2545.

25. Chao MP, Alizadeh AA, Tang C, et al. Therapeutic antibody targeting of CD47 eliminates human acute lymphoblastic leukemia. Cancer Res. 2011;71(4):13741384.

26. Zhan F, Hardin J, Kordsmeier B, et al. Global gene expression profiling of multiple myeloma, monoclonal gammopathy of undetermined significance, and normal bone marrow plasma cells. Blood. 2002;99(5):17451757

27. Yoshida K, Tsujimoto, H, Matsumura K, et al. CD47 is an adverse prognostic factor and a therapeutic target in gastric cancer. Cancer Med. 2015;4(9):1322-1333.

28. Suzuki S, Yokobori T, Tanaka N, et al. CD47 expression regulated by the miR-133a tumor suppressor is a novel prognostic marker in esophageal squamous cell carcinoma. Oncol Rep. 2012;28(2):465-472.

29. Junker A, Krumbholz M, Eisele $S$, et al MicroRNA profiling of multiple sclerosis lesions identifies modulators of the regulatory protein CD47. Brain. 2009;132(Pt 12):3342-3352.

30. Peshdary V and Atlas E. Dexamethasone induced miR-155 up-regulation in differentiating 3T3-L1 preadipocytes does not affect adipogenesis. Sci Rep. 2018;19;8(1):1264.

31. Kikuchi Y, Uno S, Kinoshita Y, et al. Apoptosis inducing bivalent single-chain antibody fragments against CD47 showed antitumor potency for multiple myeloma. Leuk Res. 2005;29(4):445-450.

32. Patel S, Wang FH, Whiteside T, Kasid U Identification of seven differentially displayed transcripts in human primary and matched metastatic head and neck squamous cell carcinoma cell lines: implications in metastasis and/or radiation response. Oral Oncol. 1997;33(3):197-203.

33. Laliberté B, Wilson AM, Nafisi $\mathrm{H}$, et al TNFAIP8: a new effector for Galpha (i) coupling to reduce cell death and induce cell transformation. J Cell Physiol. 2010; 225(3):865-874.

34. Zhang L, Liu R, Luan Y and Yao Y. Tumor necrosis factor- $\alpha$ induced protein 8: pathophysiology, clinical significance, and regulatory mechanism. Int J Biol Sci. 2018;14(4): 398-405.

35. Liu T, Gao H, Chen X, et al. TNFAIP8 as a predictor of metastasis and a novel prognostic biomarker in patients with epithelial ovarian cancer. Br J Cancer. 2013; 109(6):1685-1692.

36. Shi TY, Cheng X, Yu KD, et al. Functional variants in TNFAIP8 associated with cervical cancer susceptibility and clinical outcomes. Carcinogenesis. 2013;34(4):770-778.
37. Liu T, Xia B, Lu Y, Xu Y, Lou G. TNFAIP8 overexpression is associated with platinum resistance in epithelial ovarian cancers with optimal cytoreduction. Hum Pathol. 2014:45(6):1251-1257.

38. Eisele L, Klein-Hitpass L, Chatzimanolis N, et al. Differential expression of drug-resistance-related genes between sensitive and resistant blasts in acute myeloid leukemia. Acta Haematol. 2007;117(1):8-15.

39. Mutlu P, Ural AU, Gündüz U. Different types of cell cycle and apoptosis related gene expressions alter in corticosteroid, vincristine and melphalan resistant U-266 multiple myeloma cell lines. Turk J Hematol. 2014;31(3):231-238.

40. Amodio N, Gallo Cantafio ME, Botta C, et al. Replacement of miR-155 elicits tumor suppressive activity and antagonizes bortezomib resistance in multiple myeloma. Cancers (Basel). 2019;11:236.

41. Caracciolo D, Montesano M, Altomare E, et al. The potential role of miRNAs in multiple myeloma therapy. Expert Rev Hematol. 2018;11(10):793-803.

42. Chakraborty C, Sharma AR, Sharma G Doss CGP, Lee SS. Therapeutic miRNA and siRNA: moving from bench to clinic as next generation medicine. Mol Ther Nucleic Acids. 2017;8:132-143.

43. Ahmad N, Haider S, Jagannathan S, Anaissie E, Driscoll JJ. MicroRNA theragnostics for the clinical management of multiple myeloma. Leukemia. 2014;28(4):732-738.

44. Nana-Sinkam SP, Croce CM. Clinical applications for microRNAs in cancer. Clin Pharmacol Ther. 2013;93(1):98-104.

45. Di Martino MT, Leone E, Amodio N et al. Synthetic miR-34a mimics as a novel therapeutic agent for multiple myeloma: in vitro and in vivo evidence. Clin Cancer Res. 2012;18(22):6260-6270.

46. Rupaimoole R, Han HD, Lopez-Berestein G, and Sood AK. MicroRNA therapeutics: principles, expectations, and challenges. Chin J Cancer. 2011;30(6):368-370

47. Kwok G, Zhao TJ, Weiss J, et al Translational applications of microRNAs in cancer, and therapeutic implications. Noncoding RNA Res. 2017;2(3-4):143-150.

48. Denizli M, Aslan B, Mangala LS, et al Chitosan nanoparticles for miRNA delivery. Methods Mol Biol. 2017;1632:219-230.

49. Shah MY, Ferrajoli A, Sood AK, Lopez Berestein G, Calin GA. microRNA Therapeutics in cancer - an emerging concept. EBioMedicine. 2016;12:34-42.

50. Chen Y, Gao DY, Huang L. In vivo delivery of miRNAs for cancer therapy: challenges and strategies. Adv Drug Deliv Rev. 2015; 81:128-141 